\title{
Dual Electrochemical Quartz Crystal Microbalance with Dissipation Monitoring
}

\author{
Rafael Ecker ${ }^{1}$, Nikolaus Doppelhammer ${ }^{1}$, Bernhard Jakoby ${ }^{1}$, Erwin K. Reichel ${ }^{1}$ \\ ${ }^{1}$ Institute for Microelectronics and Microsensors, Johannes Kepler University Linz, 4040 Linz, Austria \\ rafael.ecker@jku.at, nikolaus.doppelhammer@jku.at, bernhard.jakoby@jku.at, erwin.reichel@jku.at
}

\begin{abstract}
Summary:
We report on the design and fabrication of a dual electrochemical quartz crystal microbalance sensor unit with dissipation monitoring (EQCMD). Applying an electrical field between two oppositely placed quartz surfaces facilitates particle separation and monitoring the deposition of oppositely charged particles on the electrodes during or after the colloidal synthesis of silicalite-1 zeolites. By evaluating the resonance frequencies and quality factors of the quartz crystals, several properties of the test liquid are monitored indirectly. We demonstrate the measurement of electrical conductivity and viscosity.
\end{abstract}

Keywords: EQCMD; crystallization monitoring; zeolite; viscosity measurement; fluid conductivity

\section{Introduction}

The quartz crystal microbalance with dissipation monitoring (QCMD) and the associated electrochemical version (EQCMD) are nowadays often used to detect very tiny mass changes on the surface, for example in a deposition process [1], or to characterize mechanical properties of materials [2].

The purpose of this work is to build a setup for monitoring the synthesis of zeolites. This process is based on emerging colloidal nanoparticles in an alkaline solution that grow by consuming a silicate source present as tetra(ethyl)orthosilicate (TEOS). This solution is particularly aggressive such that measurement cells have to be designed carefully including the selection of suitable materials. Besides other techniques, viscosity sensing is used to track the crystallization progress [3]. The presented setup aims at integrating the in-situ measurement of electrochemical properties and mass loading at electrodes. In particular, this setup differs from previous similar approaches by using two mass-sensitive devices facilitating the measurement of potential deposits and surface effects at each of the two electrodes used for impedance spectroscopy and viscosity sensing.

\section{Setup}

The reactor chamber is constructed as shown in Fig. 1. Top, middle and lower part are 3D printed using an Objet30 Pro 3D printer. For the fabrication of the inner chamber, castable silicone and 3D printed molds are used. This reactor chamber is surrounded by temperature- controlled aluminum housing, Peltier elements, PT100 temperature sensors, heat sinks, fans and thermal insulation.

The control task itself is executred by a Raspberry $\mathrm{PI}$ computer with additional evaluation electronics. Two quartz disk resonators operating in thickness shear mode (Tai Tien Electronic S-CAAAB-5MG03 or LapTech Precision Inc. WTiAu0514) are installed as sensors. An evaluation unit (MicroResonant QCM50) and a multiplexer are used to evaluate the resonance frequencies and the quality factors of these quartz crystals.

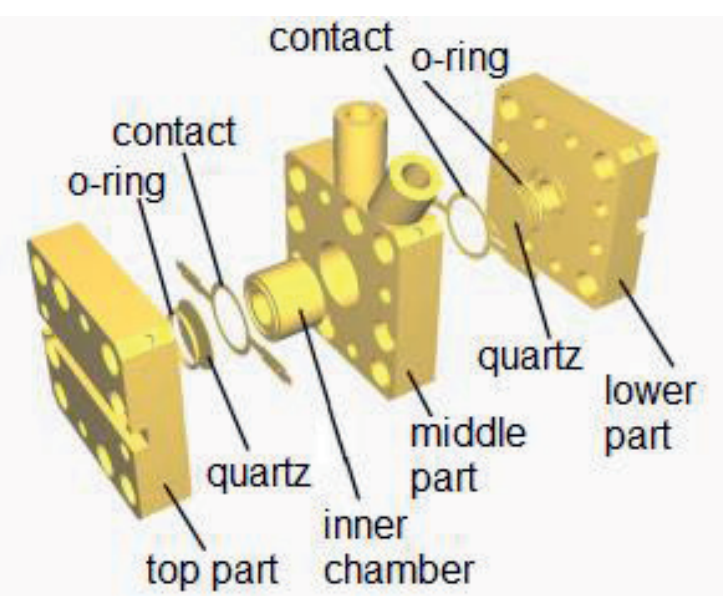

Fig. 1. Design of the reactor chamber

\section{Methods}

To determine the viscosity from the measured values of resonance frequency and quality factor, the acoustic impedance for Newtonian flu- 
ids is used [3]. This requires two reference measurements in known fluids or gases and the density of the test liquid at a known temperature.

A Reference 600+ potentiostat from Gamry Instruments is used to measure the electrical impedance of the sample between those two electrodes of the quartz sensors which are in contact with the sample. We utilized a first order model for the impedance of the liquid in terms of a resistor, modeling the ohmic resistance of the liquid, in series with a capacitance, modeling the electrochemical double layer at the electrode-liquid interface. For high frequencies the capacitance is short-circuited. Consequently, this impedance solely represents the ohmic resistance $R$ of the sample and serves as a measure for electrical conductivity.

\section{Measurements}

To test the setup, we started with viscosity and electric conductivity measurements using sodium hydroxide $(\mathrm{NaOH})$ solutions in various concentrations and compared our results to data from literature $[4,5]$. The results show that the measured values are in reasonable agreement with reference values.

Next, we did measurements monitoring the crystallization of a zeolite precursor liquid (ZPL) with a reactor temperature of $60^{\circ} \mathrm{C}$. After the first measurement we noticed that the gold electrodes of both quartz crystals, contacting the strong alkaline ZPL solution, were partly destroyed (little holes). Consequently, we covered these sides of the quartzes with a thin, about 1-2 $\mu \mathrm{m}$ thick, polymethyl methacrylate (PMMA) layer using a spin coating process. . In Fig. 2 the results with and without this protection layer are shown. Comparison of the two curves shows that the sensitivity of the quartz crystals decreases because of the PMMA layer. Otherwise both measurements show a similar course. The decreasing resonance frequency and quality factor indicates the growth of the crystals on the quartz surfaces. The reason for the regularly swings in all curves is not known yet. Measurements with the lye potassium hydroxide $(\mathrm{KOH}), 40 \%$ in water, have shown these effects too. With pure water no swings are monitored. We therefore assume that these swings are not directly related to crystallization process. But occur generally in connection with alkaline solutions. We are currently studying this phenomenon further.

\section{Conclusion}

In this paper, we presented a setup designed for the monitoring of zeolite syntheses using the principle of a dual EQCMD setup. To demonstrate its capabilities, viscosity and electrical
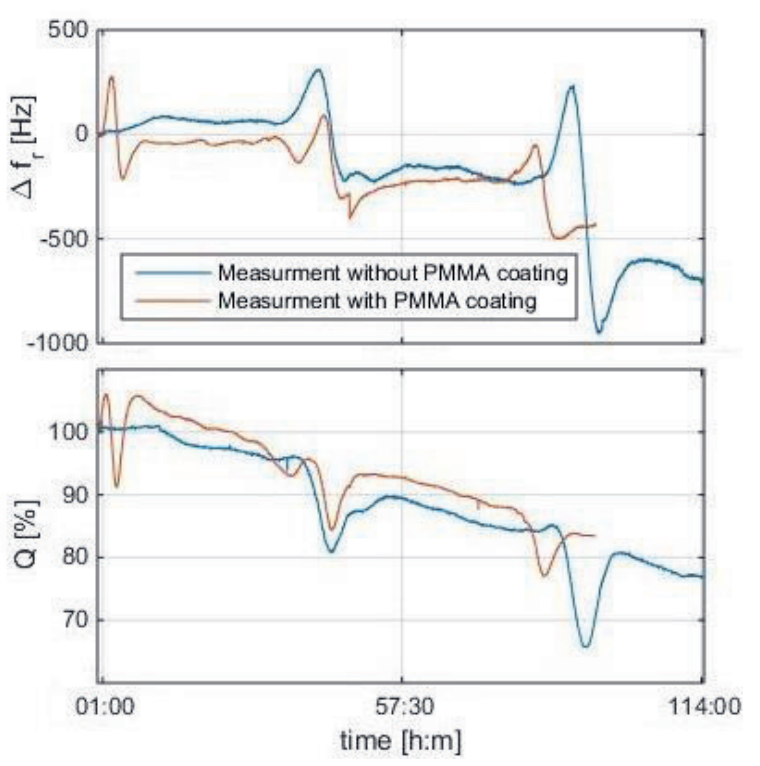

Fig. 2. Measurement result with ZPL and a reactor temperature of $60^{\circ} \mathrm{C}$ using a PMMA coated and an uncoated quartz crystal. Top graph changes in resonance frequency vs time, bottom graph quality factor vs time.

conductivity for various $\mathrm{NaOH}$ solutions are measured. We compared the results to known values from literature, where measurements agree particularly well with literature data. Our cell has the potential to monitor chemical reactions such as zeolite formation in alkaline precursor liquids or to detect other phenomena, like the regularly swings in measurement data, which we are currently investigating.

\section{References}

[1] D. Dudášová, A. Silset, J. Sjöblom, Quartz Crystal Microbalance Monitoring of Asphaltene Adsorption/Deposition, Journal of Dispersion Science and Technology 29, 139-146 (2008); doi: 10.1080/01932690701688904

[2] N. Shpigel, M.D. Levi, D. Aurbach, EQCM-D technique for complex mechanical characterization of energy storage electrodes: Background and practical guide, Energy Storage Materials 21, 399- 413(2019); doi: 10.1016/j.ensm.2019.05.026

[3] L.R. Follens, E.K. Reichel, C. Riesch, J. Vermant, J.A. Martens, C.E. Kirschhock, B. Jakoby, Viscosity sensing in heated alkaline zeolite synthesis media, Physical chemistry chemical physics 11, 2854- 2857 (2009); doi: 10.1039/B816040F

[4] P.M. Sipos, G. Hefter, P.M. May, Viscosities and Densities of Highly Concentrated Aqueous $\mathrm{MOH}$ Solutions $(\mathrm{M}+) \mathrm{Na}+, \mathrm{K}+, \mathrm{Li}+, \mathrm{Cs}+,(\mathrm{CH} 3) 4 \mathrm{~N}+)$ at 25.0 ․ J. Chem. Eng. Data 45, 613- 617 (2000); doi: $10.1021 / \mathrm{je} 000019 \mathrm{~h}$

[5] Y. Shao, M. Hellström, A. Yllö, J. Mindemark, K. Hermansson, J. Behler, C. Zhang, Temperature effects on the ionic conductivity in concentrated alkaline electrolyte solutions, Phys Chem Chem Phys. 22, 10426- 10430 (2020); doi: 10.1039/C9CP06479F 